\title{
Desenvolvimento e validação da Escala de Conflitos Intragrupos - ECIG
}

\author{
Narla Ismail Akel Silva' - Universidade de Brasilia, Brasilia, Brasil \\ Katia Puente-Palacios - Universidade de Brasilia, Brasilia, Brasil
}

\begin{abstract}
Resumo
Este artigo descreve o processo de desenvolvimento e validação da Escala de Conflitos Intragrupos (ECIG). Os itens foram formulados a partir do modelo teórico bidimensional do conflito, segundo o qual tanto as desavenças relativas às tarefas quanto as relativas às relações interpessoais compõem o construto. Foram criados inicialmente 13 itens, respondidos em escala tipo Likert de 5 pontos, que passaram por processo de validação semântica e estatística. Na validação estatística, realizada com 200 estudantes de graduação e pós-graduação de instituições pública e privada, utilizou-se a análise fatorial (PAF, rotação oblíqua). A solução mais satisfatória encontrada foi a bifatorial, com 11 itens, divididos nos fatores conflito de tarefas e de relacionamento. Ambos alcançaram índices psicométricos adequados ( $\alpha=0,82$ e $\alpha=0,86$ respectivamente) e incluíram conteúdos consistentes com o modelo teórico adotado, permitindo explicar $57,48 \%$ da variância do conflito. Implicações teóricas e práticas nas organizações, com o uso dessa escala, são discutidas.

Palavras-chave: Escala de Conflitos Intragrupos, Conflito de relacionamento, Conflito de tarefas.
\end{abstract}

\section{Development and validation of the Intragroup Conflicts Scale - ECIG}

\begin{abstract}
This article describes the development and validation process of Escala de Conflitos Intragrupos (ECIG). Item construction was based on a two dimensional theoretical model according to which intra-group conflicts construct is composed both on disagreements related to tasks and on disagreements related to interpersonal relations. Initially, 13 items were constructed using a 5-point Likert scale. These items went through a process of semantic and construct validation. The construct validation was accomplished using a factor analysis (PAF, oblique rotation) on a sample of 200 undergraduate and postgraduate students of public and private universities. The most satisfactory solution indicated two factors, one related to conflicts concerning tasks and the other related to conflicts about interpersonal relations. Both factors showed appropriate psychometric properties $(\alpha=0,82$ and $\alpha=0,86$, respectively) and their content was in accordance with the adopted theoretical model explaining $57,48 \%$ of variance of conflict. Theoretical and practical implications of the use of this scale inside organizations are discussed.

Keywords: Intragroup Conflict Scale, Interpersonal relations-related conflicts, Task-related conflicts.
\end{abstract}

No decorrer dos anos, as organizações têm demonstrado grande interesse na implantação das equipes, enquanto células de desempenho. A importância atribuída às equipes de trabalho no contexto organizacional está atrelada à visão de que indivíduos que trabalham em equipes são capazes de realizar tarefas mais complexas do que aqueles que realizam as atividades individualmente. Porém, apesar do interesse de pesquisadores por uma maior compreensão do funcionamento das equipes, existe ainda uma lacuna de estudos empíricos consistentes sobre o seu funcionamento.

Os processos grupais são evidenciados há muitos anos pela psicologia social. Dentre as teorias de destaque sobre a temática de grupo, ressalta-se a teoria de campo de Kurt Lewin (1965), que demonstra interesse na compreensão dos processos grupais existentes em diversos contextos, dentre eles indústrias e organizações. A definição de grupo em ciências

\footnotetext{
1 Agradeço ao grupo de estudo do Desempenho das Equipes DEQUI/UnB. Trabalho parcialmente financiado pela CAPES bolsa de estudo.

Endereço para correspondência: QE 30 Conj. F, Casa 43, Guará 2

71065-060 - Brasília - DF

E-mail: narlakel@gmail.com
}

sociais o considera um agregado de pessoas que trabalham formando um conjunto, mas que não necessariamente realizam suas atividades de maneira interdependente, sendo este atributo um fator fundamental para a caracterização das equipes de trabalho (Albuquerque \& Puente-Palacios, 2004)

Apesar de existirem vertentes teóricas que apontam a diferença entre grupos e equipes, neste estudo essas terminologias serão utilizadas como sinônimas, por considerar-se que equipes são grupos de trabalho que possuem características peculiares e complexas, e estão compostas por membros que compartilham objetivos, tarefas, responsabilidades e resultados (Albuquerque \& Puente-Palacios, 2004).

A definição de equipe adotada neste estudo é aquela defendida por Machado (1998), que a descreve como

um sistema de relações dinâmicas e complexas entre um conjunto de pessoas, que se identificam a si próprias e são identificadas por outras pessoas dentro da organização como membros de um grupo relativamente estável, que interagem e compartilham técnicas, regras, procedimentos e responsabilidades, utilizadas para desempenhar tarefas e atividades com a finalidade de atingir objetivos mútuos. (p. 7) 
Pode-se afirmar que a autora não define a equipe de trabalho somente pela simples junção de pessoas, mas pela relação dinâmica estabelecida entre os indivíduos, durante a execução das tarefas, sendo estas voltadas para a obtenção de um determinado objetivo compartilhado entre os membros. Autores como Sundstrom, De Meuse e Futrell (1990) assumem posições similares à adotada por Machado (1998), ao apontarem que equipes de trabalho atuam como um conjunto de indivíduos interdependentes, que compartilham objetivos e responsabilidades, por resultados específicos na organização. Assim, a presença de processos de interação social surge como aspecto central das equipes de trabalho.

O cenário atual das pesquisas, voltadas para a compreensão do funcionamento das equipes de trabalho, aponta para a necessidade de uma maior precisão ou clareza sobre os fatores que potencializam e os que restringem a sua efetividade. Estudos como o de Dimas, Lourenço e Miguez (2005) destacam que o conflito intragrupal é um fenômeno que pode vir a causar impacto na efetividade das equipes. Outros autores ainda ressaltam a importância de se considerar o impacto do conflito nos indicadores afetivos de efetividade das equipes, como a satisfação e o comprometimento (Guimarães, 2007; Jehn \& cols., 2006).

A partir dessas constatações teóricas propôs-se a realização deste trabalho, cujo principal objetivo é descrever o processo de desenvolvimento e validação da Escala de Conflitos Intragrupos (ECIG). A pesquisa traz como proposta a ampliação nacional de pesquisas e de medidas que explorem os tipos e a magnitude dos conflitos existentes nas equipes de trabalho.

Conflito é definido como um processo manifesto de incompatibilidade, desacordo ou dissonância entre entidades sociais, como pessoas, grupos ou organizações (Rahim, Antonioni, Krumov \& Ilieva, 2000). Os primeiros pesquisadores a descrever e classificar esta terminologia foram Guetzkow e Gyr (1954), ao teorizarem sobre equipes e processos decisórios e destacarem dois tipos de conflitos possíveis de emergir em um grupo de trabalho. Foram diferenciados os conflitos voltados para as tarefas que o grupo está desempenhando e o conflito baseado nas relações interpessoais no grupo, nomeando-os, respectivamente, como conflito substantivo e conflito afetivo.

A literatura internacional revelou um aumento do interesse na investigação do conflito intragrupal nas organizações de trabalho a partir dos anos 90. Em 1994, Jehn propôs uma medida que avaliasse o conflito intragrupal a partir de uma visão bidimensional, a qual continuou sendo discutida e aperfeiçoada em anos seguintes (Jehn 1994, 1997). Segundo essa autora, o conflito de relacionamento incluiria indicadores emocionais e afetivos como atritos, tensão ou raiva entre os membros do grupo, enquanto o conflito de tarefa envolveria indicadores cognitivos, como diferentes pontos de vista e opiniões relacionados ao planejamento e execução das atividades do grupo.

A visão bidimensional do conflito proposta por Jehn (1994) foi adotada por Simons e Peterson (2000), que destacam o fato de o conflito de tarefa ou conflito cognitivo estar caracterizado como a percepção de desacordos entre membros de um grupo sobre o conteúdo de suas decisões. Apontam, também, que ele envolve diferenças entre pontos de vista, ideias e opiniões em relação ao planejamento e execução das atividades. O conflito de relacionamento ou conflito emocional, segundo esses autores, seria a percepção de incompatibilidade interpessoal entre os membros. A origem desse tipo de conflito seria devida a diferenças de preferências pessoais ou políticas, de valores e de estilos interpessoais.

Jenh (1997) chega a citar um terceiro tipo, nomeado conflito intragrupal de processo. Segundo sua definição, esse tipo de conflito estaria relacionado à incompatibilidade de perspectivas ou de ideias sobre a forma de realização da tarefa, gerando divergências quanto à distribuição das funções a serem executadas pelos membros da equipe. A autora, entretanto, continuou a mensurar em seus estudos somente os dois tipos de conflito citados anteriormente, destacando que o conflito de processo estaria relacionado ao conflito de tarefa, pois diz respeito à divergência de opiniões e ideias existentes, principalmente durante a execução de tarefas complexas, que exigem maior delegação ou troca de papéis para executá-las. As evidências trazidas pelo estudo realizado por Dimas e colaboradores (2005) apontam que o conflito de processo estaria contido no conflito de tarefas, através de indicadores que denotam a sua presença, como as divergências existentes sobre a maneira de execução das tarefas e distribuição das responsabilidades.

Em estudos realizados por Simons e Peterson (2000) e Cosier, Dalton e Taylor (1991), identificou-se correlação positiva entre conflito de tarefa e conflito de relacionamento. Ou seja, quando há um conflito voltado para execução ou decisão sobre a tarefa, este também pode ser percebido como de natureza pessoal, podendo haver uma distorção sobre o real foco do conflito, vindo a impactar no surgimento do conflito de relacionamento.

Considerando as teorizações e os achados empíricos encontrados sobre esta temática, no presente estudo é adotado o modelo bidimensional de 
conflito de Jehn (1994), por haver um maior suporte empírico e conceitual na literatura pesquisada sobre o tema. Durante o processo de desenvolvimento da escala ora apresentada, compreendeu-se que o conflito de processo é inerente ao conflito de tarefas, levando em consideração também os indicadores apontados anteriormente, que remetem à sua existência (Jehn, 1997; Dimas \& cols., 2005).

Focando especificamente nas consequências do conflito, observa-se que os achados empíricos não são unânimes, tendo sido identificados efeitos tanto positivos como negativos. Assim, os resultados de pesquisas sobre a implicação dos tipos de conflito intragrupal para aqueles que o vivenciam são inconclusivos. Os conflitos intragrupais de tarefa são apontados como elementos que impactam favoravelmente na qualidade da decisão, pois a síntese que emerge da discussão demonstra a diversidade da perspectiva grupal, que geralmente é superior à perspectiva individual. O conflito de tarefa pode ser considerado como algo funcional, uma vez que pode enriquecer a compreensão entre os colegas da equipe. Conforme os membros do grupo debatem suas ideias e perspectivas, eles participam ativamente do processo grupal e, como resultado, tornam-se mais comprometidos com a decisão final (Amason, 1996).

Apesar de existirem evidências sobre o perfil funcional do conflito de tarefas, Simons e Peterson (2000) alertam para um possível caráter negativo do mesmo. Esses autores afirmam que altos níveis de conflito de tarefa percebidos no grupo de trabalho podem levar a uma redução na satisfação e no comprometimento dos membros com a equipe. Estudos de Jehn (1997) apontam que altos níveis do conflito de tarefa podem gerar interferências na conclusão destas, e que a existência de tal tipo de conflito estaria associada a um bom desempenho nas equipes que lidam com problemas que requerem soluções rápidas. No entanto, para as decisões que necessitam de mais tempo, o conflito de tarefa estaria associado com um baixo desempenho. Outros estudos revelam evidências da possibilidade de uma influência negativa do conflito de tarefa, deixando o assunto em aberto para novas pesquisas e achados (De Dreu \& Weingart, 2003).

O caráter disfuncional do conflito de relacionamento é mais evidente, pois sua ocorrência limita a habilidade dos grupos em processar corretamente a informação. Seus membros, nesse caso, focalizam o seu tempo e energia com incompatibilidades e disputas individuais, ao invés de se dedicarem aos problemas relacionados às suas tarefas (De Dreu \& Weingart, 2003). De acordo com
Jehn (1997) o conflito de relacionamento prejudica o desempenho e a satisfação dos membros do grupo.

Em relação às medidas utilizadas para mensuração do conflito intragrupal, foram encontradas, fora do Brasil, duas escalas. Jenh (1994) apresentou uma escala denominada Intragroup Conflict Scale (ICS), capaz de medir as duas dimensões do conflito propostas teoricamente, sendo eles o conflito cognitivo (tarefas) e o afetivo (relacionamento). No entanto, Pearson, Ensley e Amason (2002) apontaram que a ICS proposta por Jenh apresenta propriedades psicométricas frágeis, quando aplicada em amostras diferenciadas, prejudicando assim o poder estatístico da medida.

A Intragroup Conflict Scale foi aprimorada pelos estudos de Pearson e cols. (2002) e publicada em "nova versão", formada pela dimensão conflito de relacionamento, com 3 itens respondidos em escala tipo Likert, e pela dimensão conflito de tarefas, também constituída por três itens. Essa nova versão da ICS é uma versão reduzida da escala construída por Jehn (1994), originalmente composta por nove itens. Logo, a ICS seria constituída pelo conjunto de itens da escala de Jehn (1994) que apresentaram maior poder preditivo. $\mathrm{O}$ alfa de Cronbach encontrado para o fator conflito de relacionamento foi 0,72 e para o fator conflito de tarefas 0,77 , sendo estes bons índices de confiabilidade interna da escala segundo Tabachnick e Fidell (2007).

No Brasil, Martins, Guimarães e Oliveira (2005) construíram e validaram uma escala denominada Escala de Conflitos Intragrupais (ECI), que avalia o conflito também por meio de dois fatores. O primeiro, denominado conflito de relacionamento, composto por cinco itens, e o segundo, conflito de tarefas, contendo quatro itens. Essa escala, que apresentou índices psicométricos satisfatórios, alfa de Cronbach 0,81 para o conflito de relacionamento e 0,77 para o conflito de tarefas, foi revalidada no contexto nacional (Guimarães, 2007).

Considerando o estado da arte no país, no que diz respeito à disponibilização de ferramentas de mensuração do conflito, buscou-se prover a comunidade científica e os gestores organizacionais de uma escala validada em contexto nacional que avaliasse o conflito intragrupal. Esse é o principal motivo que justifica a realização do presente estudo, aliado à escassez de pesquisas empíricas nacionais sobre o tema. As escalas encontradas que avaliam o conflito intragrupal possuem atributos que precisam ser mais bem definidos conceitualmente ou ainda delimitados teoricamente, demandando assim, o desenvolvimento de uma nova ferramenta. 
Para o desenvolvimento da medida, foram utilizadas as escalas de Jehn (1994) e Martins, Guimarães e Oliveira (2005) como norteadoras do processo, sendo reaproveitados somente os itens que não apresentaram fragilidade semântica, como falta de clareza do conteúdo investigado, ou características de incompatibilidade com as dimensões teóricas propostas. Para um melhor entendimento desse processo, o próximo tópico descreve a forma como os itens foram construídos e validados.

\section{Método \\ Participantes}

Para atingir o objetivo proposto pela pesquisa, foram aplicados 230 questionários em uma amostra de estudantes de graduação e pós-graduação de uma universidade pública e de faculdades particulares da Capital Federal. Foram considerados somente os participantes que estivessem realizando alguma atividade laboral em equipe. Para esta investigação, foram acrescentadas perguntas adicionais, a fim de se obterem respostas sobre a natureza do trabalho realizado. O conjunto de participantes era composto, em sua maioria, de homens (55\%) e a média de idade foi de 25 anos $(\mathrm{dp}=10)$. Além disso, $72 \%$ eram estudantes de graduação e $28 \%$ cursavam pósgraduação.

\section{Instrumento}

\section{Construção e validação semântica da escala}

Os itens foram construídos com base nas escalas de conflito intragrupais já existentes (Jehn, 1994; Martins \& cols., 2005). As contribuições teóricas que embasaram a construção dos itens também foram obtidas de campos relativos aos indicadores cognitivos e afetivos dos dois tipos de conflito abordados, sendo estes o conflito de tarefas (base cognitiva) e o conflito de relacionamento (base afetiva) (Jehn, 1994; 1997; Simons \& Peterson, 2000; Dimas \& colaboradores, 2005).

Durante o processo de desenvolvimento da medida, dois itens foram retirados da Intragroup Conflict Scale, sendo estes os que denotam a existência de raiva e conflito emocional entre os membros. Outros quatro itens foram retirados da escala de Martins e cols. (2005) e reformulados. Os conteúdos desses itens remetem aos indicadores de desacordo sobre as decisões de trabalho, tensão emocional no relacionamento interpessoal, diferença de opinião na realização das tarefas e discordância sobre a maneira de execução das tarefas. Novos itens foram construídos, sendo sete no total. As descrições desses itens remetem aos indicadores atrito pessoal, diferença de opinião no planejamento das tarefas, desacordo pessoal, conflito durante delegação das atividades, tempo gasto na resolução de conflitos interpessoais, desafeto entre os membros e conflito durante realização do trabalho.

A primeira versão da Escala de Conflito Intragrupal ficou composta por 13 itens, que foram submetidos, em um primeiro momento, a uma validação semântica com intuito de verificar a adequação da redação proposta. Sua compreensão foi testada por 10 pessoas com perfis semelhantes aos dos possíveis respondentes deste estudo, que leram os itens e discutiram sua clareza e pertinência semântica. Este processo resultou na reformulação de quatro itens, a fim de se atingir uma melhor adequação entre itens e dimensões teóricas propostas.

\section{Análise de concordância dos itens}

$\mathrm{O}$ conjunto de treze itens foi submetido à avaliação de sete juízes, pesquisadores na área de psicologia organizacional. Foram apresentadas as definições dos dois tipos de conflitos a serem avaliados pela medida e solicitado que cada item fosse classificado na dimensão correspondente. Para cada um dos itens calculou-se o índice de concordância (IC) entre os julgamentos, sendo que deveriam ser descartados os itens com IC $<80 \%$ (Pasquali, 1997, 1999). Adotando este critério, não foi necessário o descarte de qualquer item, somente uma pequena reformulação na redação de dois deles.

A escala aplicada neste estudo denomina-se Escala de Conflitos Intragrupos (ECIG), composta por 13 itens que descrevem o que ocorre na atual equipe de trabalho. Os itens são respondidos em escala tipo Likert de intensidade de 5 pontos, variando de 1 (nenhum) a 5 (muitíssimo). O instrumento é autoaplicável, sendo as instruções de respostas descritas no cabeçalho. A medida em questão, conforme descrito, originou-se de escalas preexistentes, cujos itens serviram de base para a pesquisa. Da escala de Jehn (1994) aproveitaram-se dois itens, sendo o conteúdo dos mesmos voltado para a existência de raiva e tensão entre os membros. Em relação ao instrumento de Martins e cols. (2005), quatro itens foram tomados como ponto de partida, tendo seu conteúdo ajustado às bases teóricas adotadas para o estudo. Esses itens remetiam a questões voltadas para divergências ou discordâncias em relação à execução das atividades. Também foram criados novos itens, contemplando questões como o tempo gasto pela equipe para a resolução de conflitos interpessoais e diferenças de opinião sobre o planejamento das atividades. 


\section{Procedimento}

O instrumento foi aplicado por uma dupla de pesquisadoras em estudantes universitários trabalhadores formais ou informais que julgavam realizar seu trabalho em equipe. Previamente à coleta de dados propriamente dita, os possíveis respondentes foram informados pelo pesquisador a respeito $\mathrm{da}$ natureza da pesquisa da qual iriam participar. Esclareceu-se que as respostas dadas não teriam qualquer relação com o desempenho acadêmico na instituição em que estudavam e que os questionários eram anônimos. Logo, não precisariam se identificar e suas participações eram voluntárias. Assim, somente foram coletados dados daqueles que desejaram cooperar com a pesquisa que lhes fora brevemente apresentada. A coleta ocorreu em sala de aula e o tempo estimado de resposta da escala foi em torno de 10 minutos. Foram descartados trinta questionários, de participantes que não se consideraram como pertencentes a uma equipe de trabalho. O número total de questionários válidos para o estudo foi de 200 , indicador suficiente para conduzir a análise estatística, sendo obtidos acima de 10 casos por item (Hair, Anderson, Tatham \& Black, 2005).

\section{Análise de dados}

Os dados obtidos foram analisados inicialmente através de procedimentos de estatística descritiva, com o objetivo de verificar a distribuição das respostas por itens de forma a se obterem conclusões sobre a normalidade da sua distribuição. A adequação e fatorabilidade da amostra foram testadas mediante cálculo do índice de Kaiser-Meyer- Olkin (KMO), da inspeção da matriz de correlações dos itens e do teste de esfericidade de Bartlett, conforme apontado por Tabachnick e Fidell (2007). Posteriormente, os dados foram submetidos à análise dos eixos principais (PAF). Uma vez identificada a solução fatorial mais satisfatória, foi verificada a confiabilidade interna de cada fator, por meio do cálculo do alfa de Cronbach e da média aritmética da correlação item-total.

\section{Resultados}

A inspeção da matriz de correlações revelou a existência de mais de 50\% dos casos (itens) com coeficientes significativos e superiores a 0,30 (entre 0,35 e 0,64), havendo a indicação da fatorabilidade da matriz. A esse respeito Laros (2005) manifesta que os itens constitutivos da escala, que não revelam certo índice de correlações significativas entre si, poderão acarretar uma pobre contribuição na estrutura fatorial, visto que não compartilham da mesma variância. A seguir, a medida de adequação da amostra foi calculada e apresentou um excelente resultado de $\mathrm{KMO}=0,93$, mostrando ser pertinente a redução dos itens a fatores. $\mathrm{O}$ teste de esfericidade de Bartlett apontou também a pertinência de buscar a redução do conjunto de itens a um número menor de fatores.

Confirmada a pertinência de fatorar matriz de dados, foram adotados procedimentos com vistas a identificar o número mais adequado de fatores a serem apreendidos. A estimativa do número possível de fatores foi feita mediante avaliação de três indicadores matemáticos: análise dos eigenvalues (valores próprios), gráfico scree plot e análise paralela. Além disso, levou-se em consideração o fato de a escala ser oriunda de um modelo teórico que propõe uma estrutura bifatorial do conflito intragrupal. Logo, analisou-se também a convergência teórica do resultado do processo.

A análise utilizando o critério dos eigenvalues maiores ou iguais a um, conforme proposto por Guttman (1954) e Kaiser (1960), mostrou que a matriz de dados sob análise poderia suportar até dois fatores. A análise visual do gráfico scree plot também identificou a existência de dois fatores. A análise paralela baseada na comparação entre os valores de eigenvalues obtidos na amostra e os valores aleatórios, calculados a partir do número de casos e do número de itens, indicou a existência de somente um fator.

Para a extração dos fatores, os itens foram forçados a se separarem em dois conjuntos, uma vez que o arcabouço teórico em que o presente trabalho se sustenta defende a existência de dois tipos de conflito. Esta decisão converge também com alguns dos critérios matemáticos adotados.

Foi utilizada, para a extração dos fatores, a análise fatorial - PAF (Principal Axis Factoring), considerando-se a possibilidade da existência de uma solução bifatorial. A rotação utilizada para a verificação da solução foi a direct oblimim em razão da expectativa de relação entre os fatores. Como critério de contribuição mínima do item no fator, foi adotado o valor de 0,32 , seguindo recomendações oferecidas por Tabachnick e Fidell (2007).

Uma vez extraídos dois fatores e analisada a contribuição (carga fatorial) de cada item, a análise do conteúdo dos itens agrupados foi realizada. Ela mostrou que a solução fatorial mais satisfatória era, de fato, a composta por dois fatores. Vale lembrar que tão importante quanto a aplicação de critérios matemáticos é a verificação da pertinência teórica do conjunto de itens no fator proposto, conforme o destacam Laros e Puente-Palacios (2004).

A análise da solução fatorial, levando em consideração tanto os critérios matemáticos quanto os teóricos, evidenciou 2 itens com contribuições pobres. 
A análise da adequação teórica da solução revelou que um item que indagava sobre a existência de conflito relativo à realização do trabalho enquadrava-se no fator conflito de relacionamento, enquanto a literatura indica o provável pertencimento deste ao fator conflito de tarefas. Logo, mesmo apresentando carga de 0,44, foi excluído da medida por não haver sentido teórico o enquadramento deste no fator proposto pela análise estatística. De maneira adicional, observou-se que o item que aborda a existência de conflito emocional entre os membros apresentou cargas significativas (acima de 0,30) nos dois fatores. Por haver uma diferença menor do que 0,1 entre as duas cargas, esse item também acabou por ser excluído da medida (Tabachnick \& Fidell, 2007), tendo em vista a falta de especificidade do conteúdo abordado pelo item, que enquadrou-se tanto no conflito de tarefas quanto no conflito de relacionamento.
Com o conjunto composto por 11 itens agregados em dois fatores, obteve-se $57,48 \%$ da variância total explicada pela solução adotada. Após as decisões tomadas quanto à composição de cada fator, procedeu-se à análise da confiabilidade interna de cada, utilizando-se para tanto o cálculo da correlação itemtotal e o alfa de Cronbach. No caso do fator 1, composto por 5 itens e denominado Conflito de Tarefas, o valor do alfa foi 0,82 e a média aritmética correlação item-total 0,66 . No caso do fator 2, composto por seis itens e denominado Conflito de Relacionamento, esses valores foram 0,86 e 0,66, respectivamente. Com base na magnitude dos índices calculados para investigar a confiabilidade interna dos fatores da Escala de Conflito Intragrupal, é pertinente afirmar que a medida cumpre o objetivo para o qual foi desenvolvida, qual seja, medir a magnitude do conflito existente em equipes de trabalho. Um resumo desses dados encontra-se apresentado na Tabela 1.

Tabela 1 - Características psicométricas dos fatores da Escala de Conflitos Intragrupos

\begin{tabular}{|c|c|c|c|}
\hline Fatores & Item & Carga fatorial & $\mathrm{h}^{2}$ \\
\hline \multirow[t]{2}{*}{ 1. Conflito de tarefas } & Desacordo/decisões & 0,54 & 0,52 \\
\hline & Opinião/planejamento & 0,56 & 0,38 \\
\hline Alfa de Cronbach $=0,82$ & Opinião/realização & 0,84 & 0,57 \\
\hline \multirow[t]{2}{*}{ Correlação item-total $=0,66$} & Conflito/delegação & 0,64 & 0,50 \\
\hline & Discordância/execução & 0,65 & 0,52 \\
\hline \multirow[t]{3}{*}{ 2. Conflito de relacionamento } & Atrito/ pessoal & 0,62 & 0,52 \\
\hline & Tensão/ emocional & 0,59 & 0,48 \\
\hline & Desacordo/ pessoal & 0,86 & 0,61 \\
\hline Alfa de Cronbach $=0,86$ & Raiva & 0,78 & 0,58 \\
\hline \multirow[t]{2}{*}{ Correlação item-total $=0,66$} & Tempo/interpessoais & 0,58 & 0,37 \\
\hline & Desafeto & 0,72 & 0,53 \\
\hline
\end{tabular}

Total de variância explicada $=57,48 \%$

Finalmente, considerando que o método de rotação escolhido para a extração dos fatores foi oblimin, logo, pressupõe correlação entre eles, escores fatoriais foram calculados para permitir essa constatação. $\mathrm{O}$ valor da correlação entre os fatores foi 0,72 , confirmando, dessa forma, a pertinência da escolha dessa rotação e evidenciando que o conflito de tarefas não é independente do conflito de relacionamento, pois entre eles ocorre um compartilhamento de $51,84 \%$ da variância.

As implicações dos achados apresentados nesta seção serão discutidas na seguinte, de maneira a explorar tanto o seu significado e suas implicações teóricas quanto a utilidade prática para os gestores organizacionais.

\section{Discussão}

Considerando os dois tipos de conflito intragrupal apontados por Simons e Peterson (2000) e
De Drew e Weingart (2003), os resultados da escala desenvolvida apresentam convergência com o suporte teórico em que se sustentam. Os achados empíricos deste estudo evidenciam que a Escala de Conflitos Intragrupos (ECIG) é similar à Intragroup Conflict Scale (ICS) construída por Jehn (1994), no que tange à estrutura fatorial ou na quantidade de dimensões encontradas. Assim, é pertinente concluir que o trabalho ora relatado pode ser considerado um passo inicial de desenvolvimento de uma medida de conflito aplicável à realidade brasileira. Trata-se, portanto, de uma ferramenta de diagnóstico do conflito em equipes de trabalho.

Apesar dos resultados da análise paralela terem apontado para solução unifatorial, a solução mais satisfatória foi a bifatorial. A esse respeito cabe comentar que os critérios matemáticos não podem superar os critérios teóricos. A pesquisa realizada com apoio de análises psicométricas adota critérios 
matemáticos cujo peso é o mesmo dado às análises teóricas. Laros e Puente-Palacios (2004) destacam que os modelos teóricos não podem ser guiados por números. É imprescindível que exista um corpo teórico robusto em que qualquer procedimento matemático estará sustentado. Assim, a solução bifatorial é a que consegue capturar parte significativa do fenômeno que pretende mensurar.

Há de se observar que, apesar da forte correlação percebida entre os dois tipos de conflito, a literatura aponta a diferenciação existente entre ambos (Amason, 1996; Guimarães, 2007; Jehn, 1997; Simons e Peterson, 2000). A existência de correlação entre os dois fatores foi indiciada por Amason (1996); Cosier, Dalton e Taylor (1991) e Simons e Peterson (2000), ao sugerirem que os tipos de conflitos intragrupais coexistem.

Em relação à natureza de cada fator, os dados obtidos tomando por base a solução fatorial identificada evidenciam a especificidade de cada um. O primeiro fator foca no conflito de tarefas, e os itens que o constituem descrevem divergências ou tensão no grupo, decorrentes de atividades como tomada de decisões, planejamento das atividades, realização do trabalho, delegação e execução. Assim, observa-se que, conforme defendido por Jehn (1994), o foco desse tipo de conflito recai sobre as atividades que podem ser vistas como trabalho da equipe.

O segundo fator, denominado conflito de relacionamento, está composto por um conjunto de seis itens, os quais abordam questões relativas à vida interpessoal dos membros. Toca em assuntos como atrito pessoal, tensão emocional, desacordos, sentimentos de raiva entre os membros e desafetos. Portanto observa-se como o foco desse núcleo consegue se restringir aos limites teóricos definidos pelos autores da área (Pearson \& cols., 2002; Dimas \& colaboradores, 2005; Jehn, 1994; 1997; Martins \& cols., 2005; Simons \& Peterson, 2000).

Pela constatação da pertinência teórica e das propriedades psicométricas da medida desenvolvida, é adequado manifestar que se trata de instrumento útil para o diagnóstico da existência de conflito nas equipes de trabalho, destacando-se o fato de permitir identificar se ele é de natureza laboral (conflito de tarefas) ou relacional. A importância desse diagnóstico está em permitir aos gestores organizacionais conhecer a especificidade do conflito vivenciado nas equipes para, com base nele, procurar fazer a gestão mais adequada.

\section{Considerações finais}

A partir dos resultados estatísticos aqui apresentados, parece pertinente concluir que a Escala de Conflitos Intragrupos é um instrumento válido para a mensuração desse fenômeno no contexto dos grupos ou equipes de trabalho. Trata-se de uma ferramenta a ser utilizada por pesquisadores e gestores na exploração e no diagnóstico do conflito que emerge entre os membros de uma mesma equipe de trabalho. Recomenda-se a realização de outros estudos que permitam verificar a estabilidade da estrutura bifatorial identificada. Sugere-se que novas pesquisas sejam desenvolvidas em outros contextos, de preferência em organizações de trabalho que possuam como valor real o trabalho em equipe.

Um dos propósitos desta pesquisa foi oferecer um instrumento que confirmasse o modelo teórico de estudos anteriores, visando à mensuração de fenômenos que ocorrem no contexto das organizações, mais precisamente nos cenários das equipes. A realização de um estudo dessa natureza poderá favorecer pesquisas futuras sobre modelos explicativos do conflito intragrupal.

Este estudo demonstra que a linha que separa o conflito de tarefas do conflito de relacionamento é tênue. $\mathrm{O}$ conhecimento acerca dos tipos de conflitos e de suas características é necessário a uma adequada gestão de equipes. Cabe aos membros da equipe e ao supervisor guiar suas ações, por exemplo, privilegiando processos internos que evidenciem mais aspectos cognitivos como a troca ou discussão de ideias, opiniões ou soluções voltadas para as tarefas, em detrimento de processos que evidenciem aspectos emocionais/ afetivos entre os membros, como raiva, tensão ou intolerância.

Apesar de o conflito intragrupal ser, na maioria das vezes, definido pelas abordagens tradicionais como algo negativo ou prejudicial, devendo, sempre que possível, ser evitado pela equipe de trabalho, é preciso considerar os tipos de conflitos existentes e suas diferentes predições e efeitos (Dimas \& cols., 2005; Jehn, 1997). O conflito é um processo natural e inerente à dinâmica organizacional e das equipes. Pode emergir por meio das relações entre indivíduos de um mesmo grupo ou equipe, entre grupos ou equipes, entre os diferentes níveis organizacionais e entre organizações. Novas respostas a questões levantadas em estudos sobre a presente temática virão a contribuir para o esclarecimento do fascinante e às vezes obscuro mundo do conflito intragrupal. 


\section{Referências}

Albuquerque, F. J. B. \& Puente-Palacios, K. E. (2004). Grupos e equipes de trabalho nas organizações. Em J. C. Zanelli, J.E. Borges-Andrade \& A.V.B. Bastos (Orgs.). Psicologia, organizações e trabalho no Brasil (pp. 357-379). Porto Alegre: Artmed.

Amason, A. C. (1996). Distinguishing the effects of functional and dysfunctional conflict on strategic decision making: resolving a paradox for top management teams. Academy of Management Journal, 39, 123-149.

Cosier, R. A., Dalton, D. R. \& Taylor, L. A. (1991). Positive effects of cognitive conflict and employee voice. Employee Responsibilities and Rights Journal, 4(1), 7-11.

De Dreu, C. \& Weingart, L. R. (2003). Task versus relationship conflict, team performance and team member satisfaction: a meta analysis. Journal of Applied Psychology, 88(4), 741-749.

Dimas, I. D., Lourenço, P. R. \& Miguez, J. (2005). Conflitos e desenvolvimento grupal: em direção a uma abordagem integrada. Psychologica, 38, 103119.

Guetzkow, H. \& Gyr, J. (1954). An analysis of conflict in decision-making groups. Human Relations, 7, 367-382.

Guimarães, V. F. (2007). Bases de poder do supervisor, conflitos intragrupais e comportamento organizacional $e$ com a equipe: um estudo exploratório. Dissertação de Mestrado. Universidade Federal de Uberlândia, MG.

Guttman, L. (1954). Some necessary conditions for common factor analysis. Psychometrika, 19, 149162.

Hair, J. F. Anderson, R. E. Tatham, R. L. \& Black, W. (2005). Análise multivariada de dados. Porto Alegre: Bookman.

Jehn, K. A. (1994). Enhancing effectiveness: an investigation of advantages and disadvantages of valued based intragroup conflict. International Journal of Conflict Management, 5, 223-238.

Jehn, K. A. (1997). A qualitative analysis of conflict types and dimensions in organizational groups. Administrative Science Quarterly, 42, 530-558.

Jehn, K. A, Rupert, J. \& Nauta, A. (2006). The effects of conflict asymmetry on mediation outcomes: satisfaction, work motivation and absenteeism. International Journal of Conflict Management, 17(2), 96109.

Kaiser, H. F. (1960). The application of electronic computers to factor analysis. Educational and Psycological Measurement, 20, 141-151.

Laros, J. A. (2005). O uso da análise fatorial: algumas diretrizes para pesquisadores. Em L. Pasquali. (Org.). Análise fatorial para pesquisadores (pp. 163184). Brasília: LabPAM.

Laros J. A. \& Puente-Palacios K. E. (2004). Validação cruzada de uma escala de clima social. Estudos de Psicologia (Natal), 9(1), 113-119.

Lewin, K. (1965). Teoria de Campo em ciência social. Trad. de Carolina Martuscelli Bori. São Paulo: Pioneira.

Martins, M. C. F., Guimarães, V. F. \& Oliveira, M. C. (2005). Adaptação e validação fatorial da escala de conflitos intragrupais. Trabalho apresentado no II Congresso Brasileiro de Psicologia Organizacional e do Trabalho, Brasília, DF.

Pasquali, L. (1997). Psicometria: teoria e aplicaşões. Brasília: Editora da UnB.

Pasquali, L. (1999) (Org.). Instrumentos psicológicos: manual prático de elaboração. Brasília: LABPAM/IBAPP.

Pearson, A. W., Ensley, M. D. \& Amason, A. C. (2002). An assessment and refinement of Jehn'n intragroup conflict scale. International Journal of Conflict Management, 13(2), 110-126.

Rahim, M. A., Antonioni, D., Krumov, K. \& Ilieva, S. (2000). Power, conflict and effectiveness: a crosscultural study in the United States and Bulgaria. European Psychologist, 5(1), 28-33.

Simons, T. \& Peterson, R. (2000). Task conflict and relationship conflict in top management teams: the pivotal role of intragroup trust. Journal of Applied Psychology, 85(1), 102-111.

Sundstron, E., De Meuse, K. \& Futrell, D. (1990). Work teams: applications and effectiveness. American Psychologist, 45, 120-133.

Tabachnick, B. \& Fidell, L. (2007). Using multivariate statistics. Boston: Pearson.

Recebido em setembro de 2009

Reformulado em janeiro de 2010 Aprovado em março de 2010 
Sobre as autoras:

Narla Ismail Akel Silva é psicóloga graduada pelo Centro Universitário de Brasília (UniCeub) e mestre em Psicologia do Trabalho e das Organizações pelo PG/PSTO/UnB. Atualmente é docente do Instituto de Educação Superior de Brasilia (IESB). Dentre os temas de interesse destacam-se Medidas em Psicologia e Comportamento Organizacional.

Katia Puente-Palacios é doutora em Psicologia pela UnB, professora da mesma universidade e atualmente trabalha como docente do Curso de Pós-Graduação em Psicologia Social, do Trabalho e das Organizações (PG/PSTO/UnB). Tem como tema de pesquisa o estudo da efetividade de equipes de trabalho e os seus preditores, adotando para isto modelos matemáticos. 